\title{
Proteome-Scale Profiling Reveals MAFG and MAFF as Two Novel Candidate Key Transcription Factors Involved in Palmitic acid Induced Umbilical Vein Endothelial Cells Apoptosis
}

\section{Mangyuan Wang}

Xinjiang Medical University Affiliated First Hospital

Fen Liu

Xinjiang Medical University Affiliated First Hospital

\section{Binbin Fang}

Xinjiang Medical University Affiliated First Hospital

Qiang Huo

Xinjiang Medical University Affiliated First Hospital

Yining Yang ( $\nabla$ yangyn5126@163.com )

Xinjiang Medical University https://orcid.org/0000-0002-8332-8508

\section{Research article}

Keywords: Apoptosis, atherosclerosis, endothelial cell, HUVEC, palmitic acid, transcription factor

Posted Date: September 25th, 2020

DOI: https://doi.org/10.21203/rs.3.rs-72835/v1

License: (c) (1) This work is licensed under a Creative Commons Attribution 4.0 International License.

Read Full License 


\section{Abstract}

Backgrounds: Vascular endothelial cell apoptosis is the first risk factor of atherosclerosis (AS), and it can be induced by high doses of glucose and palmitic acid (PA). The purpose of our study is to use a new generation of high-throughput transcription factors (TFs) detecting method to identify novel candidate key TFs involved in PA-induced vascular endothelial cell apoptosis.

Methods: Human umbilical vein endothelial cells (HUVECs) were treated with $0 \mu \mathrm{M}$ PA (control group), $250 \mu \mathrm{M}$ PA (group 1), or 500 $\mathrm{M}$ PA (group 2). Candidate TFs among the three groups were determined by significant changes according to t-test, and pathway enrichment, western blot (WB) and RT-qPCR were then performed.

Results: Fifty-one TFs showing with significant $p$ value were identified, and 24 TFs with significant $p$ value plus fold change $>2$ and with dose-dependence were identified with 12 TFs biologically validated in former studies. Two of the remaining 12 novel TFs, v-maf musculoaponeurotic fibrosarcoma oncogene family protein $\mathrm{G}$ (MAFG) and $\mathrm{v}$-maf musculoaponeurotic fibrosarcoma oncogene family protein $\mathrm{F}$ (MAFF), were matched to AS known signalling pathways and were validated by WB and RT-qPCR in our study.

Conclusions: We identified MAFG and MAFF as novel candidate key TFs in vascular endothelial cell apoptosis, which is the key initial process of AS.

\section{Background}

Atherosclerosis (AS) is a common vascular disease characterized by AS plaque formation, which initiates with endothelial cell apoptosis, followed by lipid deposition and foam-cell formation (1). AS is the leading cause of cardiac infarction, arterial aneurysm, or stroke, and the severe associated complications (2).

Vascular endothelial cell apoptosis is the initial pathogenetic process underlying AS plaque formation, and it is the first risk factor of AS plaque formation (1). To interfere effectively in early stage of AS or to predict AS plaque formation risk, we focus on identifying the key transcription factors (TFs) in this initial process of AS formation. Human umbilical vein endothelial cell (HUVEC) is a cell line that recapitulates the characteristics of arterial endothelial cells in vitro. HUVEC apoptosis induced by 24-hour stimulation with palmitic acid (PA) represents a mature AS cell model (3). Numerous TFs and signalling pathways, as well as changes in gene expression levels, are likely to be involved in HUVEC apoptosis (4).

TF response element (TFRE) profiling is a new generation of high-throughput TFs detecting method that allows qualitative and semi-quantitative screening of TFs on a proteomic scale in specific cells or tissues, using nano-liquid chromatography/tandem mass spectrometry (LC-MS) analysis (5). TFRE profiling provides information that directly reflects changes in TF protein levels as well as the activity state of the TF populations. 
Although previous studies have reported some TFs that are key players in HUVEC apoptosis (6), highthroughput TF profiling in HUVEC apoptosis models has not yet been reported.

In this study, to identify key candidate TFs involved in HUVEC apoptosis, we conducted TFRE profiling using three experimental groups of HUVECs. In this study, to identify key candidate TFs involved in HUVEC apoptosis, we conducted TFRE profiling using three experimental groups of HUVECs, treated with or without increasing concentrations of PA and glucose to induce apoptosis.

\section{Methods}

\subsection{Experimental groups, workflow, and study design of TFRE profiling}

The HUVECs were grouped as follows: control group, HUVECs treated with $0 \mu \mathrm{M}$ PA; group 1, HUVECs stimulated with $250 \mu \mathrm{M}$ PA; experimental group 2, HUVECs stimulated with $500 \mu \mathrm{M}$ PA. All three groups were treated with PA and glucose for 24 hours, then TFRE profiling experiments were performed three times.

\subsection{HUVEC culture}

HUVECs were purchased from COBIOER company (Nanjing, China), and the designated type is primary cell from Promocell. The cells have been checked to ensure they are free of contamination, and they have been used from young stock. We then cultured the cells in Xinjiang Key Laboratory of Cardiovascular Disease. HUVECs were seeded into 18 culture flasks $\left(25 \mathrm{~cm}^{2}\right.$ in size), of which six flasks were used for each of the three experimental groups. For each group, the six flasks then were divided into two flasks, which were considered as one replicate; the experiment was therefore conducted with three replicates.

\subsection{TFRE experiment}

TFRE profiling was performed in three major steps: nuclear protein extraction, TF enrichment in nuclear protein extracts using concatenated tandem array of the consensus TFREs (catTFREs), and SDS-PAGE and in-gel digestion. The detailed procedures are described in our previous study(7).

\subsection{LC-MS analysis for protein identification and label-free quantification}

LC-MS analysis for protein identification and label-free quantification were performed as previously described $(7,8)$.

\subsection{Selection of candidates for key TFs of HUVEC apoptosis}

The frequency of total (FOT)-adjusted peak areas were compared among the three groups, and heatmap and volcano plots were drawn based on TF showing variations with both t-test $P$ value $<0.05$ and fold- 
change $>2$. Then, a relational network among these TFs was generated to find the central ones. Next, we selected discrepant TFs showing the same variation trends among the three experimental groups.

Downstream gene pathway enrichment analysis of the discrepant TFs with the same variation trends was conducted to select TFs matching to known signalling pathways in AS, which were selected as candidate TFs involved in HUVEC apoptosis. Then, we excluded known key TFs involved in AS in others' former studies, and the remaining TFs were selected as candidate novel TFs involved in HUVEC apoptosis after Western Blot (WB) and Real-time quantitative PCR (RT-qPCR) verification.

\subsection{WB and RT-qPCR}

Antibodies for WB were purchased from Abcam (Anti-MAFG: ab86524. Anti-MAFF: ab183859), and primers for RT-qPCR were synthesized as Table 1 shown. Process of WB and RT-qPCR had been described in our former study(9)

Table 1

Primers using in RT-qPCR assay

\begin{tabular}{|lll|}
\hline Molecules & Forward & Reverse \\
\hline MAFG & TCCAGGGTACTGACCTGCTC & GTTTCCTTTATTGGGGGTCG \\
\hline MAFF & TGCCCAGGTCCCATTTCTC & GGCCCACGAAGGGAATGT \\
\hline -actin & CCTAGAAGCATTTGCGGTGG & GAGCTACGAGCTGCCTGACG \\
\hline
\end{tabular}

\subsection{Statistical analysis}

Statistical analysis was performed as described previously (7).

\section{Results}

\subsection{Identification of 51 TFs showing significant differential expression ( $P<0.05$ and fold-change $>2$ )}

Among the three TFRE profiling experiments, we identified 173 TFs totally in control group, group 1 , and group 2. Of these, we identified 36 TFs, namely ATF3, HOXA5, GATA6, HOXA9, HOXB4, ALX1, ELK3, RARB, TOX2, STAT1, STAT6, TTF1, ATF7, MAFG, FOXP1, NFE2L1, RFX5, NR2F1, TCF7L2, CBFB, FOSL1, NFKB2, FOXF1, TFDP1, HMGB1, HMGB3, FLI1, RELA, RBPJ, ARID1A, HOXD8, MAFF, UBTF, HMGB2, E2F4, and FOX01, showing significant differential expression, with t-test $P$ value $<0.05$, between group 1 and control group, and identified 34 TFs, namely ATF3, HOXA5, XBP1, HOXA9, HOXB4, ALX1, ELK3, RARB, TOX2, STAT1, STAT6, AEBP1, TTF1, ATF7, FOXP1, NFE2L1, AHR, NOC3L, NR2F1, CBFB, JUNB, FOSL1, PBX2, TFDP1, MAX, HMGB1, NFAT5, FLI1, RBPJ, ARID1A, HOXD8, JUN, E2F4, and FOX01, with t-test P value < 0.05 between group 2 and control group, and identified 13 TFs, namely ATF3, XBP1, ELF2, AEBP1, NFE2L1, RFX5, NFKB2, HMG20B, NFATC2, RELA, MGA, PDS5B, and NFATC1, with t-test $P$ value $<0.05$ between group 2 and group 1 . All the 51 significant TFs, namely ATF3, HOXA5, GATA6, XBP1, HOXA9, 
HOXB4, ALX1, ELK3, RARB, TOX2, ELF2, STAT1, STAT6, AEBP1, TTF1, ATF7, MAFG, FOXP1, NFE2L1, AHR, RFX5, NOC3L, NR2F1, TCF7L2, CBFB, JUNB, FOSL1, NFKB2, HMG20B, PBX2, FOXF1, TFDP1, MAX, HMGB1, HMGB3, NFAT5, FLI1, NFATC2, RELA, MGA, RBPJ, ARID1A, HOXD8, PDS5B, MAFF, UBTF, HMGB2, JUN, NFATC1, E2F4, and FOX01, are shown in Fig. 1A.

As is shown in the volcano plots (Fig. 1B-1D), 34 TFs significantly different between group 1 and control group were identified and met the selection criteria of both $P$ value $<0.05$ and fold-change $>2$ (Fig. $1 B$ ). Of these, 11 TFs, namely ATF3, GATA6, STAT1, STAT6, MAFG, FOXP1, NFE2L1, RFX5, TCF7L2, CBFB, and MAFF, were up-regulated, shown as red dots in Fig. 1B, and 23 TFs, namely HOXA5, HOXA9, HOXB4, ALX1, ELK3, RARB, TOX2, TTF1, ATF7, NR2F1, FOSL1, NFKB2, FOXF1, TFDP1, HMGB1,HMGB3, FLI1, RELA, RBPJ, UBTF, HMGB2, E2F4, and FOX01, were down-regulated, shown as blue dots in Fig. $1 \mathrm{~B}$. Thirteen TFs showing significantly different levels between group 2 and group 1 met the selection criteria (Fig. 1C), of which 8 TFs, namely ATF3, XBP1, STAT6, NFE2L1, NFKB2, NFATC2, RELA, and NFATC1, were up-regulated (red dots) and 5 TFs, namely ELF2, RFX5, HMG20B, MGA, and PDS5B, were down-regulated (blue dots). Finally, 33 TFs showing significant differential expression between group 2 and control group met the selection criteria (Fig. 1D); of these, 12 TFs, namely ATF3, XBP1, STAT1, STAT6, AEBP1, FOXP1, NFE2L1, NOC3L, CBFB, JUNB, NFAT5, and HOXD8, were up-regulated (red dots) and 21 TFs, namely HOXA5, HOXA9, HOXB4, ALX1, ELK3, RARB, TOX2, TTF1, ATF7, AHR, NR2F1, FOSL1, PBX2, TFDP1, MAX, HMGB1, FLI1, RBPJ, ARID1A, JUN, and E2F4, down-regulated (blue dots).

\subsection{Protein interaction network analysis and significant TFs increasing or decreasing between control group and group 1 and between group 1 and group 2 with dose dependence}

Protein interaction network analysis (Fig. 2A) revealed that several TFs were significantly changed and interconnected, including ATF3, JUN, JUNB, MAFG, MAFF, RELA, STAT1, and STAT6. Among the TFs showing differential expression from control group to group 1 and from group 1 to group 2 with dose dependence, 12 of the 51 significant TFs were up-regulated from control group to group 1 and still from group 1 to group2 (Fig. 2B), namely ATF3, XBP1, STAT1, STAT6, AEBP1, MAFG, NFE2L1, CBFB, JUNB, NFAT5, HOXD8, and MAFF, while 12 were down-regulated from control group to group 1 and still from group 1 to group2 (Fig. 2C), namely HOXA5, ALX1, ELK3, RARB, ATF7, AHR, PBX2, MAX, FLI, ARID1A, JUN, and E2F4. Therefore, these 12 up-regulated TFs and 12 down-regulated TFs were dose-dependent. Seven TFs were overlapping between the significant TFs in the interaction network analysis result and the significantly changed TFs from control group to group 1 and from group 1 to group 2 with dose dependence: ATF3, JUN, JUNB, MAFG, MAFF, STAT1, and STAT6.

\subsection{Pathway enrichment of TFs and matching to key signalling pathways of AS and WB and RT-qPCR validation}

In order to identify which of the 7 TFs is correlated with known AS pathways to further shrink the range of TFRE screening result for novel candidate key TFs in AS onset, We performed downstream gene pathway enrichment analysis of the 7 overlapping TFs (Fig. 3), and 4 TFs were matched to known key signalling 
pathways of AS, namely NFE2L2 (Nrf2)-ARE, NFKB, and MAPK signalling pathway. The analysis identified four TFs (Fig. 3C, 3E, 3F, 3G): JUN (AP1), MAFG, MAFF, STAT1.

We identified four candidate TFs, JUN (AP1), MAFG, MAFF, STAT1, in the former Sect. 3.3., and the two of the four TFs, JUN and STAT1, were then found as two known key TFs in AS in others' former studies by searching articles $(10,11)$. Therefore, the rest two novel candidate key TFs, MAFG and MAFF, were verified by performing WB and RT-qPCR. The results of RT-qPCR (Fig. 4A, 4B) and WB (Fig. 4C, 4D, 4E, 4F) showed the changing trends of these two TFs were consistent with the changing trends in TFRE results, so MAFG and MAFF were verified as two novel candidate key TFs in AS.

\section{Discussion}

\subsection{Major findings}

Here, we identified 51 TFs that showed significant differential expression, with t-test $P$ value $<0.05$ and fold-change $>2$. Then, seven overlapping TFs between the significantly regulated and connected TFs and the differentially expressed TFs from control group to group 2 with dose dependence were identified, four of which were found to have downstream genes matching to known signalling pathways of AS. The two of the four TFs, MAFG and MAFF, were still unknown in AS, then WB and RT-qPCR verification results demonstrated these two TFs were two novel candidate key TFs in AS.

\subsection{Significance of this study}

Numerous TFs are involved in vascular endothelial cell apoptosis, which is the key process underlying AS onset. However, a proteome-scale transcriptional profiling to identify candidate TFs associated with this key event in AS pathogenesis has never been performed. Our results revealed several novel candidate key TFs by proteomic profiling with high-throughput TF expression analysis. Manipulating the expression of these TFs will allow biological validation of the candidates in the future. Finally, if some of the candidates are validated in the future, these novel TFs involved in vascular endothelial cell apoptosis will be useful for early-stage medical intervention on AS.

\subsection{Comparison with other relevant studies and deductions from major results}

Several studies have reported key TFs in AS, which were validated through observing changes in AS plaque area and thickness following manipulation of the TF gene expression in an ApoE-/- mouse AS model $(6,12-29)$. In this study, we also identified some of these key TFs, such as ATF3 and JUN (AP1). In addition, ALX1, ELK3, RARB, ATF7, MAFG, NFE2L1, CBFB, PBX2, MAX, FLI1, ARID1A, and MAFF were newly identified TFs reported in our present study as candidate key TFs in AS.

According to the Genome Informatics database, the 24 known and unknown TFs identified here are all involved in AS-related biological processes, such as cell proliferation and apoptosis. Our results showed that 4 candidate TFs were up-regulated from control group to group 2 with dose dependence. Considering 
that the concentration of PA goes from 0 to $500 \mu \mathrm{M}$ from control group to group 2, this indicates that the upregulation of the 4 TFs from control group to group 2 suggests a relevant association with vascular endothelial cell apoptosis, making them attractive targets for early therapeutic intervention. The key signalling pathways of AS on which current research has focused are NFE2L2-ARE, MAPK, and NFKB pathway $(30,31)$. In addition, pathway enrichment for downstream genes of JUN (AP1), MAFG, MAFF, and STAT1 showed association with key AS signalling pathways. However, JUN (AP1) and STAT1 had already been biologically validated as key TFs in AS in a previous study $(10,11)$. Therefore, we deducted from our results that MAFG and MAFF might play a key role in HUVEC apoptosis via the NFE2L2-ARE signalling pathway because MAFG and MAFF pathway enriched to NFE2L2, which is the key molecule of NFE2L2-ARE signalling pathway. For this reason, we speculate that MAFG and MAFF are two candidate novel TFs in vascular endothelial cell apoptosis.

The function of v-maf musculoaponeurotic fibrosarcoma oncogene family protein $G$ (MAFG) and v-maf musculoaponeurotic fibrosarcoma oncogene family protein $F$ (MAFF) are now known as proliferation and differentiation coactivators collaborated with NFE2L2 in mice esophagus and forestomach keratinocyte in Keap1-NFE2L2 pathway(32), and the protein MAFG collaborates with NFE2L2 to transcriptionally activate MAFG gene through an antioxidant response element (ARE) in 293T cells(33). The protein MAFG also promotes the differentiation of mice megakaryocytes to platelets(34).

The study of Motohashi $\mathrm{H}$, et al(32) demonstrated MAFG and MAFF collaboratively interact with NFE2L2 to play key role in mice keratinocyte proliferation and differentiation IN THE Keap1-NFE2L2 pathway, and the study of Katsuoka F, et al(33) revealed NFE2L2/MAFG heterodimer binds to ARE to activate the antioxidation by oxidative stress in 293T cells. However, NFE2L1 is significantly increased between control group and experimental groups instead of NFE2L2 in our study accompanied significant increase of MAFG and MAFF. Therefore, we speculate that MAFG, MAFF, and NFE2L1 can transcriptionally activates ARE associated genes collaboratively in arterial endothelial cells, then the expression of these ARE associated genes lead to antioxidation enhancement of arterial endothelial cells. Antioxidation enhancement and cells injury reduction of arterial endothelium play a crucial role in anti-atherogensis.

\subsection{Limitations}

First, TFRE profiling is a semi-quantitative method with limited accuracy. Second, the candidate novel TFs have not been validated experimentally, although this could be achieved in a future study.

\section{Conclusion}

Here, we identified two novel candidate key TFs, MAFG and MAFF, involved in PA + glucose-induced vascular endothelial cell apoptosis, which is a key event underlying onset of AS. These candidates will need to be biologically validated in a PA-induced HUVEC apoptosis model and further in ApoE-/- mouse AS model in the future.

\section{Abbreviations}


AS, atherosclerosis

HUVEC, human umbilical vein endothelial cell

PA, palmitic acid

TFs, transcription factors

TFRE, TF response element

LC-MS, nano-liquid chromatography/tandem mass spectrometry

MAFG, v-maf musculoaponeurotic fibrosarcoma oncogene family protein $G$

MAFF, v-maf musculoaponeurotic fibrosarcoma oncogene family protein $\mathrm{F}$

WB, western blot

RT-qPCR, Real-time quantitative PCR

\section{Declarations}

\section{Ethics approval and consent to participate}

No human studies were carried out by the authors for this article. No animal studies were carried out by the authors for this article.

\section{Consent to publish}

Not applicable

\section{Availability of data and materials}

Not applicable

\section{Competing interests}

None

\section{Funding}

This work was supported by the Postdoctoral Research Foundation of China. These funding bodies did not play any role in the design of the study and collection, analysis, and data interpretation and manuscript writing.

\section{Authors' contributions}


MW designed the study, did experiments, and wrote the manuscript. FL did experiments. BF did experiments. QH and YY designed the study. All authors have read and approved the manuscript.

\section{Acknowledgements}

We would like to thank Dr. Lei Song and Mingwei Liu for their support with the TFRE experiments.

\section{References}

1. Kasikara C, Doran AC, Cai B, Tabas I. The role of non-resolving inflammation in atherosclerosis. J Clin Investig. 2018;128(7):2713-23.

2. Benjamin EJ, Virani SS, Callaway CW, Chamberlain AM, Chang AR, Cheng S, et al. Heart Disease and Stroke Statistics-2018 Update: A Report From the American Heart Association. Circulation. 2018;137(12):e67-492.

3. Dagher Z, Ruderman N, Tornheim K, Ido Y. Acute regulation of fatty acid oxidation and amp-activated protein kinase in human umbilical vein endothelial cells. Circulation research. 2001;88(12):1276-82.

4. Marin EP, Derakhshan B, Lam TT, Davalos A, Sessa WC. Endothelial cell palmitoylproteomic identifies novel lipid-modified targets and potential substrates for protein acyl transferases. Circulation research. 2012;110(10):1336-44.

5. Ding C, Chan DW, Liu W, Liu M, Li D, Song L, et al. Proteome-wide profiling of activated transcription factors with a concatenated tandem array of transcription factor response elements. Proc Natl Acad Sci USA. 2013;110(17):6771-6.

6. Niu N, Xu S, Xu Y, Little PJ, Jin ZG. Targeting Mechanosensitive Transcription Factors in Atherosclerosis. Trends Pharmacol Sci. 2019;40(4):253-66.

7. Wang M, Hu S, Nie Y, Song J. Proteomic profiling of key transcription factors in the process of neonatal mouse cardiac regeneration capacity loss. Cell biology international. 2019.

8. Feng J, Ding C, Qiu N, Ni X, Zhan D, Liu W, et al. Firmiana: towards a one-stop proteomic cloud platform for data processing and analysis. Nature biotechnology. 2017;35(5):409-12.

9. Song J, Wang M, Chen X, Liu L, Chen L, Song Z, et al. Prolactin mediates effects of chronic psychological stress on induction of fibrofatty cells in the heart. American journal of translational research. 2016;8(2):644-52.

10. Partridge J, Carlsen H, Enesa K, Chaudhury H, Zakkar M, Luong L, et al. Laminar shear stress acts as a switch to regulate divergent functions of NF-kappaB in endothelial cells. FASEB journal: official publication of the Federation of American Societies for Experimental Biology. 2007;21(13):3553-61.

11. Wang Y, Han Z, Fan Y, Zhang J, Chen K, Gao L, et al. MicroRNA-9 Inhibits NLRP3 Inflammasome Activation in Human Atherosclerosis Inflammation Cell Models through the JAK1/STAT Signaling Pathway. Cellular physiology and biochemistry: international journal of experimental cellular physiology, biochemistry, and pharmacology. 2017;41(4):1555-71. 
12. Gold ES, Ramsey SA, Sartain MJ, Selinummi J, Podolsky I, Rodriguez DJ, et al. ATF3 protects against atherosclerosis by suppressing 25-hydroxycholesterol-induced lipid body formation. The Journal of experimental medicine. 2012;209(4):807-17.

13. Nazari-Jahantigh M, Wei Y, Noels H, Akhtar S, Zhou Z, Koenen RR, et al. MicroRNA-155 promotes atherosclerosis by repressing Bcl6 in macrophages. J Clin Investig. 2012;122(11):4190-202.

14. Joly AL, Seitz C, Liu S, Kuznetsov NV, Gertow K, Westerberg LS, et al. Alternative Splicing of FOXP3 Controls Regulatory T Cell Effector Functions and Is Associated With Human Atherosclerotic Plaque Stability. Circulation research. 2018;122(10):1385-94.

15. Li K, Ching D, Luk FS, Raffai RL. Apolipoprotein E enhances microRNA-146a in monocytes and macrophages to suppress nuclear factor-kappaB-driven inflammation and atherosclerosis. Circulation research. 2015;117(1):e1-11.

16. Chen HH, Keyhanian K, Zhou X, Vilmundarson RO, Almontashiri NA, Cruz SA, et al. IRF2BP2 Reduces Macrophage Inflammation and Susceptibility to Atherosclerosis. Circulation research. 2015;117(8):671-83.

17. Sayin VI, Khan OM, Pehlivanoglu LE, Staffas A, Ibrahim MX, Asplund A, et al. Loss of one copy of Zfp148 reduces lesional macrophage proliferation and atherosclerosis in mice by activating p53. Circulation research. 2014;115(9):781-9.

18. Guo Y, Fan Y, Zhang J, Lomberk GA, Zhou Z, Sun L, et al. Perhexiline activates KLF14 and reduces atherosclerosis by modulating ApoA-I production. J Clin Investig. 2015;125(10):3819-30.

19. Hsieh J, Koseki M, Molusky MM, Yakushiji E, Ichi I, Westerterp M, et al. TTC39B deficiency stabilizes LXR reducing both atherosclerosis and steatohepatitis. Nature. 2016;535(7611):303-7.

20. Gage MC, Becares N, Louie R, Waddington KE, Zhang Y, Tittanegro TH, et al. Disrupting LXRalpha phosphorylation promotes FoxM1 expression and modulates atherosclerosis by inducing macrophage proliferation. Proc Natl Acad Sci USA. 2018;115(28):E6556-e65.

21. Zhuang T, Liu J, Chen X, Zhang L, Pi J, Sun H, et al. Endothelial Foxp1 Suppresses Atherosclerosis via Modulation of NIrp3 Inflammasome Activation. Circulation research. 2019.

22. Mahmoud MM, Kim HR, Xing R, Hsiao S, Mammoto A, Chen J, et al. TWIST1 Integrates Endothelial Responses to Flow in Vascular Dysfunction and Atherosclerosis. Circulation research. 2016;119(3):450-62.

23. Dunn J, Qiu H, Kim S, Jjingo D, Hoffman R, Kim CW, et al. Flow-dependent epigenetic DNA methylation regulates endothelial gene expression and atherosclerosis. J Clin Investig. 2014;124(7):3187-99.

24. Sage AP, Nus M, Bagchi Chakraborty J, Tsiantoulas D, Newland SA, Finigan AJ, et al. X-Box Binding Protein-1 Dependent Plasma Cell Responses Limit the Development of Atherosclerosis. Circulation research. 2017;121(3):270-81.

25. Lim WS, Timmins JM, Seimon TA, Sadler A, Kolodgie FD, Virmani R, et al. Signal transducer and activator of transcription-1 is critical for apoptosis in macrophages subjected to endoplasmic 
reticulum stress in vitro and in advanced atherosclerotic lesions in vivo. Circulation. 2008;117(7):940-51.

26. Xu L, Cheng D, Huang Z, Ding S, Zhang W, Tan H, et al. Histamine promotes the differentiation of macrophages from $\mathrm{CD} 11 \mathrm{~b}(+)$ myeloid cells and formation of foam cells through a Stat6-dependent pathway. Atherosclerosis. 2017;263:42-52.

27. Majdalawieh A, Zhang L, Fuki IV, Rader DJ, Ro HS. Adipocyte enhancer-binding protein 1 is a potential novel atherogenic factor involved in macrophage cholesterol homeostasis and inflammation. Proc Natl Acad Sci USA. 2006;103(7):2346-51.

28. Bock KW. Human AHR functions in vascular tissue: Pro- and anti-inflammatory responses of AHR agonists in atherosclerosis. Biochem Pharmacol. 2019;159:116-20.

29. Halterman JA, Kwon HM, Leitinger N, Wamhoff BR. NFAT5 expression in bone marrow-derived cells enhances atherosclerosis and drives macrophage migration. Frontiers in physiology. 2012;3:313.

30. Pan JX. LncRNA H19 promotes atherosclerosis by regulating MAPK and NF-kB signaling pathway. Eur Rev Med Pharmacol Sci. 2017;21(2):322-8.

31. Chen B, Lu Y, Chen Y, Cheng J. The role of Nrf2 in oxidative stress-induced endothelial injuries. J Endocrinol. 2015;225(3):R83-99.

32. Motohashi H, Katsuoka F, Engel JD, Yamamoto M. Small Maf proteins serve as transcriptional cofactors for keratinocyte differentiation in the Keap1-Nrf2 regulatory pathway. Proc Natl Acad Sci USA. 2004;101(17):6379-84.

33. Katsuoka F, Motohashi H, Engel JD, Yamamoto M. Nrf2 transcriptionally activates the mafG gene through an antioxidant response element. J Biol Chem. 2005;280(6):4483-90.

34. Shavit JA, Motohashi H, Onodera K, Akasaka J, Yamamoto M, Engel JD. Impaired megakaryopoiesis and behavioral defects in mafG-null mutant mice. Genes Dev. 1998;12(14):2164-74.

\section{Figures}


A

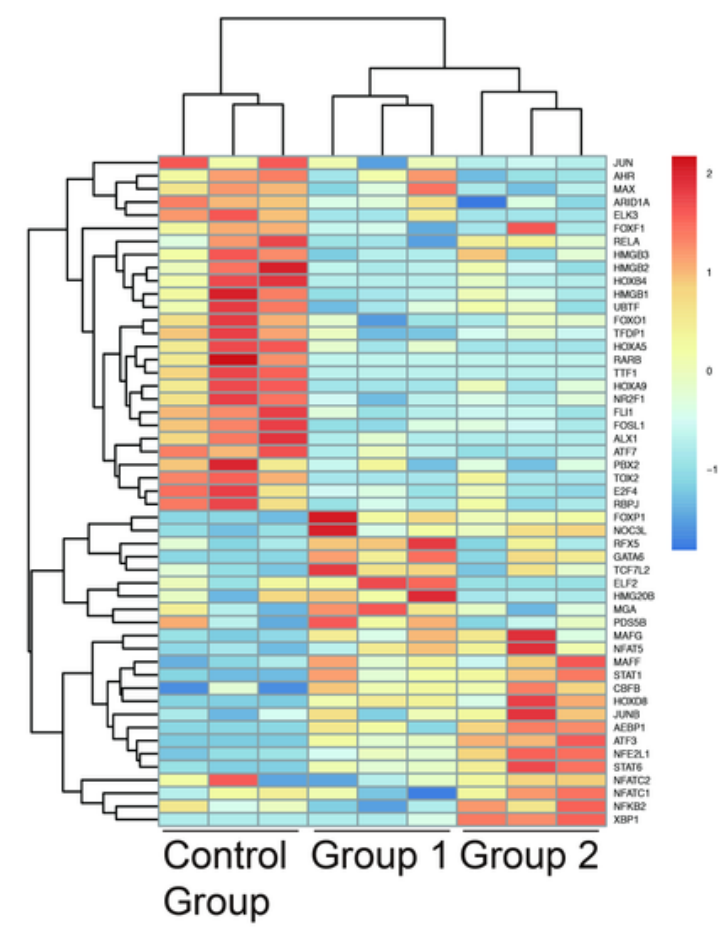

C

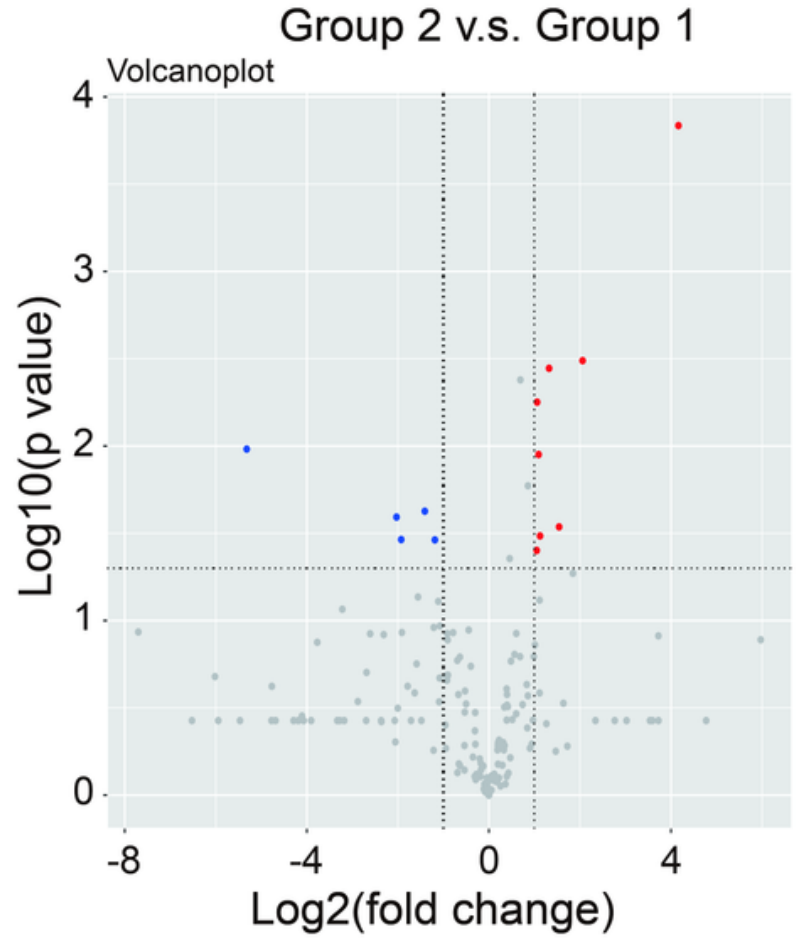

\section{B}

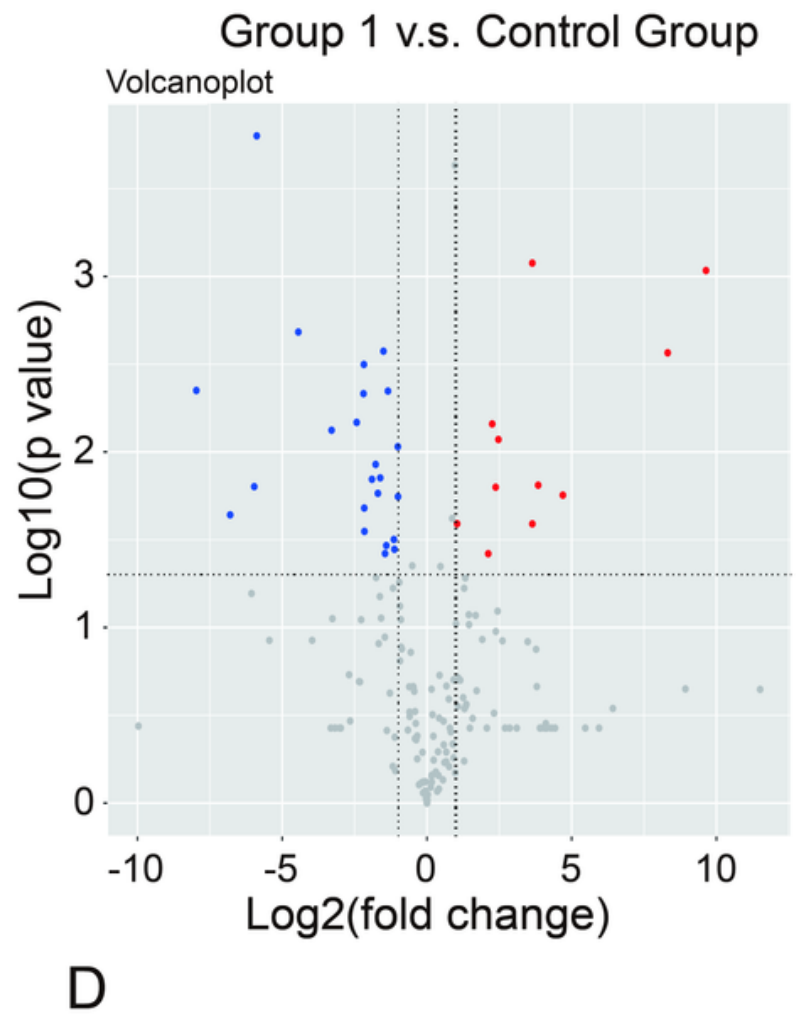

Volcanoplot

Group 2 v.s. Control Group

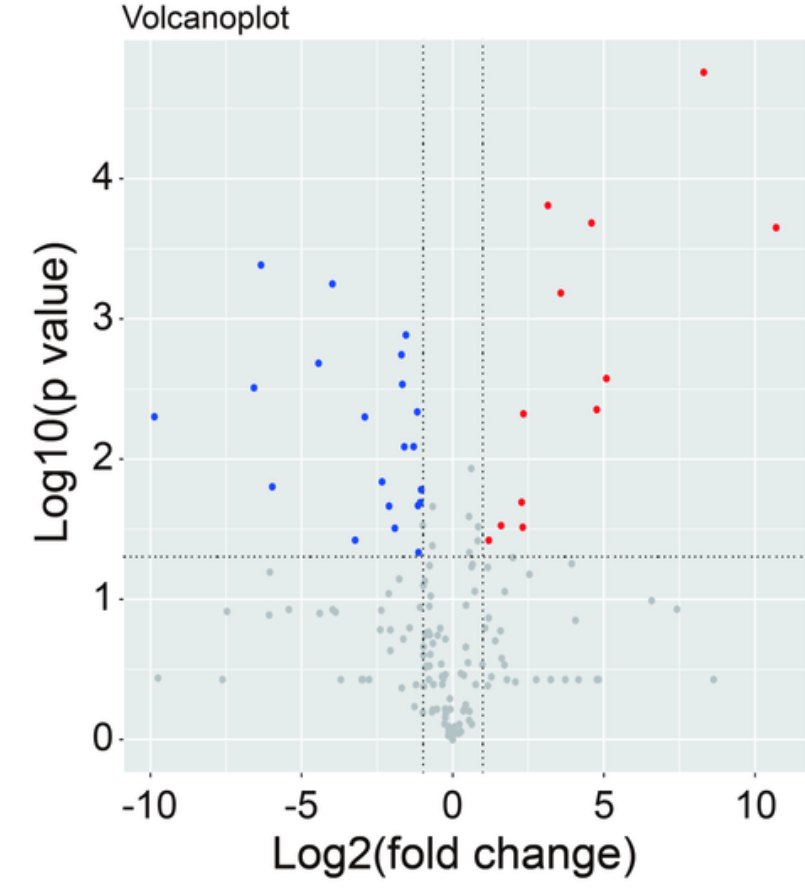

\section{Figure 1}

Identification of 51 significant TFs with $t$-test $P$ value $<0.05$ and fold-change $>2$. (A) The heatmap shows 51 transcription factors (TFs) that showed significant differential expression among three experimental groups. Blue and red represent low and high expression level, respectively. (B, C, D) Each dot in the volcano plots shows one TF with both fold-change $>2$ and $P$ value in t-test $<0.05$. The red dots represent 
up-regulated transcription factors, and the blue ones represent down-regulated transcription factors, respectively, between two of the three groups.
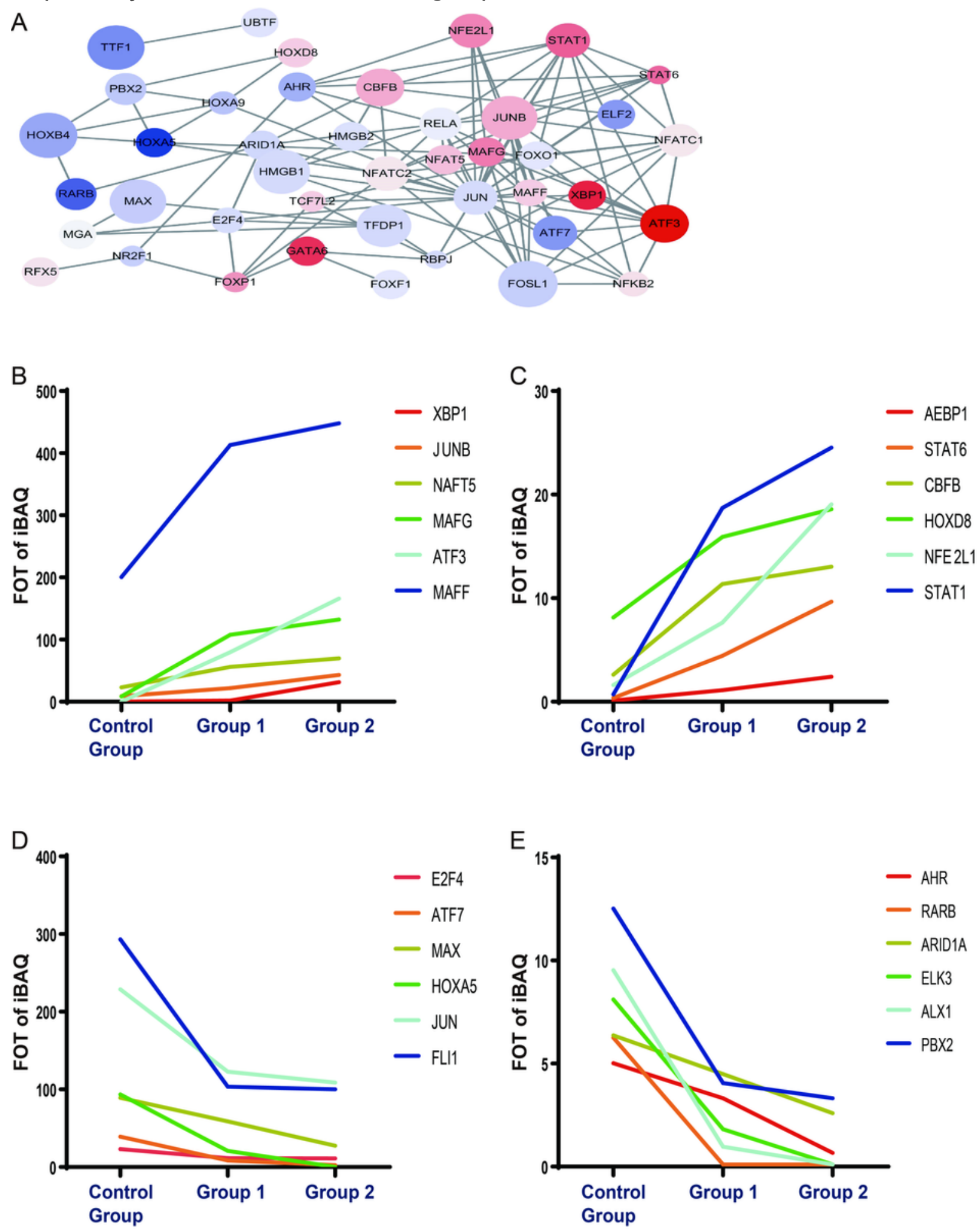

Figure 2

Protein interaction network analysis and discrepant TFs increasing or decreasing from control group to group 2. (A) Relational network among the 51 transcription factors that showed significant differential expression among three experimental groups. The area of the circle represents the number of interactions 
of the transcription factor. Red and blue represent up-regulation and down-regulation, respectively. (B, C, D, E) Twenty-four discrepant TFs increasing or decreasing from control group to group 2.
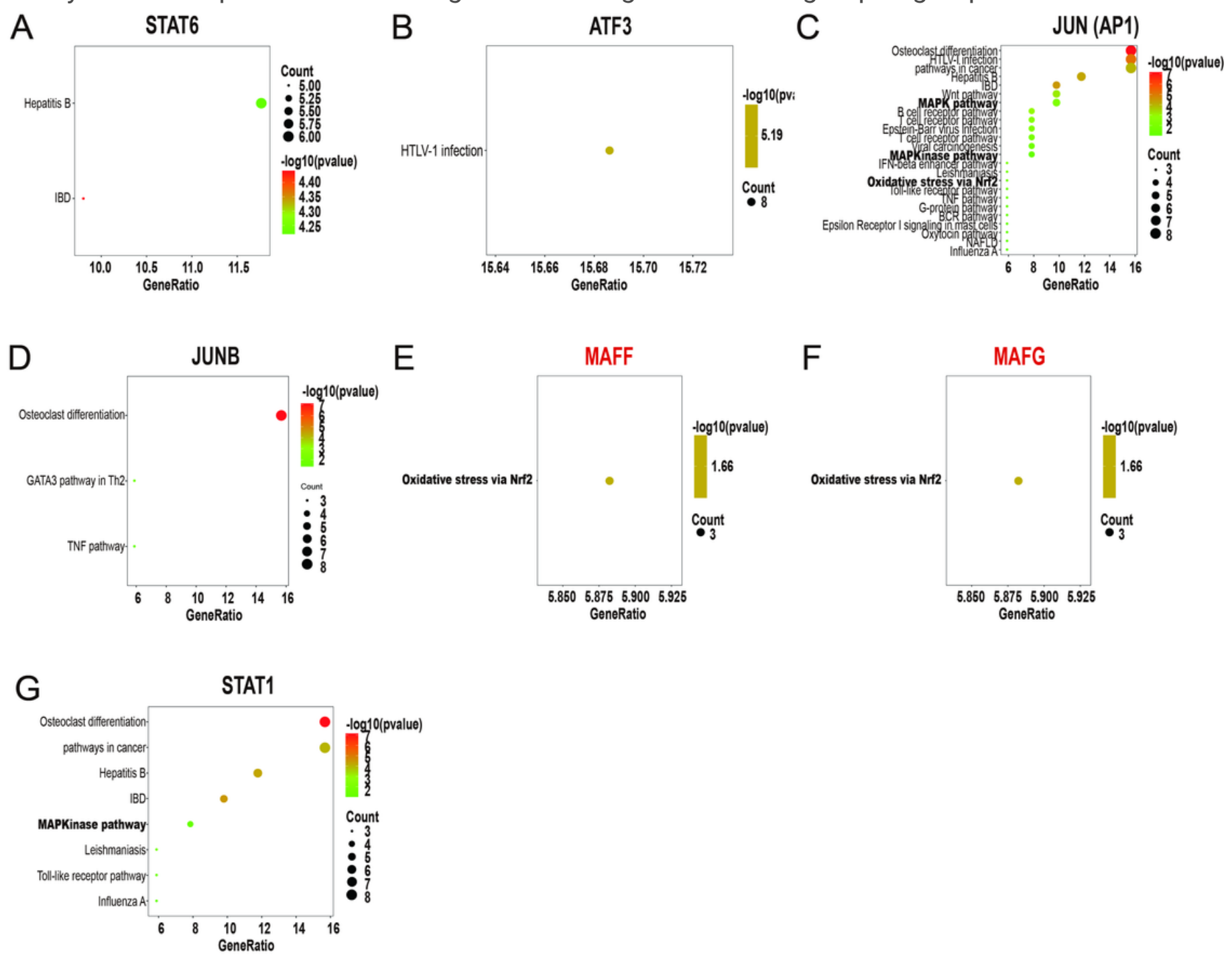

\section{Figure 3}

Pathway enrichment of TF downstream genes and matching to key signalling pathways of AS or vascular endothelial cell apoptosis. Signalling pathways with bold letters are known key signalling pathways of atherosclerosis. The transcription factors indicated with red letters are the novel candidate key TFs involved in vascular endothelial cell apoptosis. 


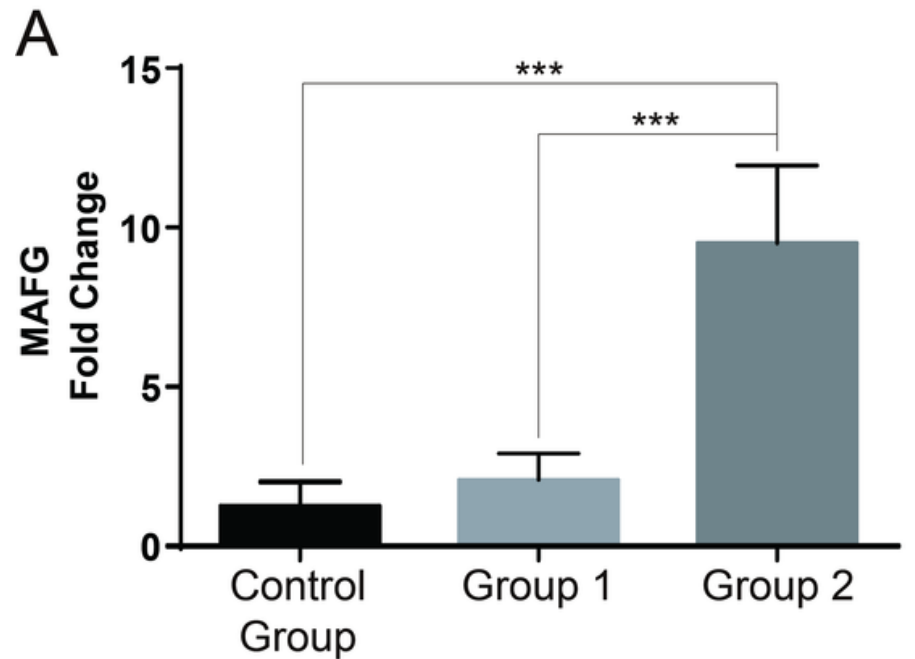

C
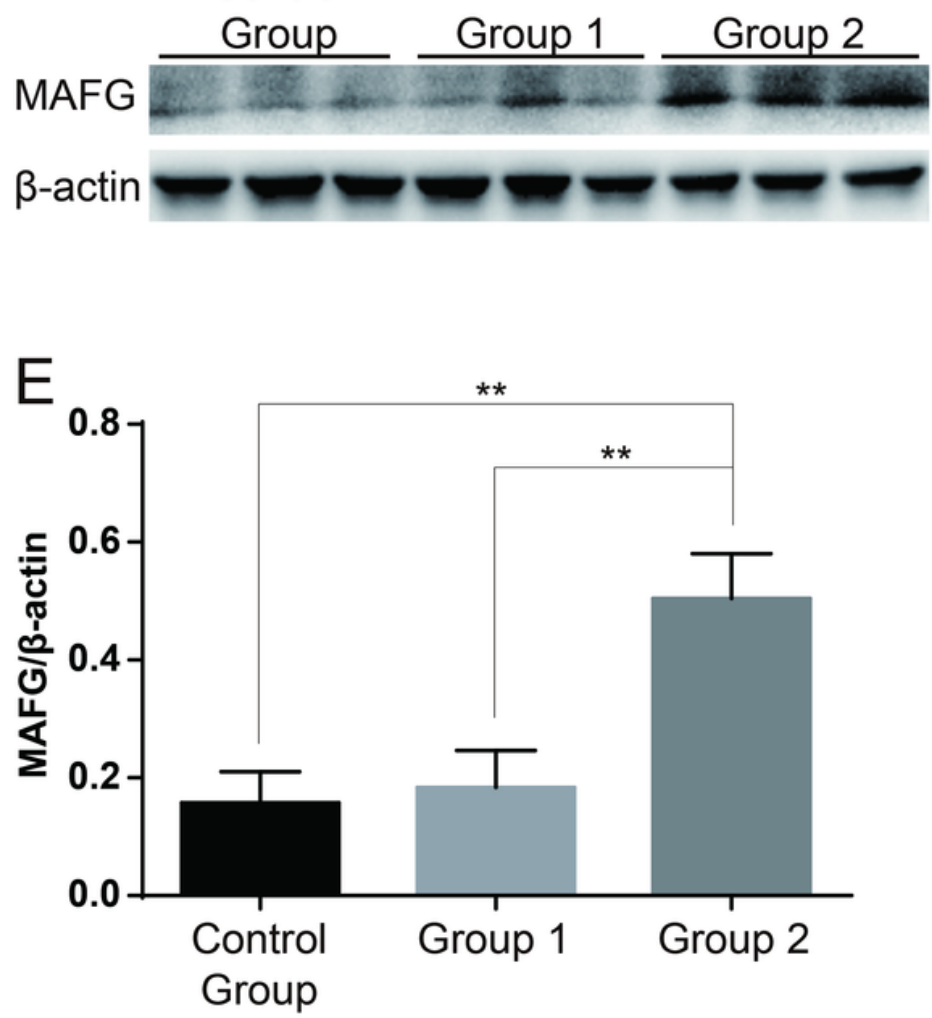

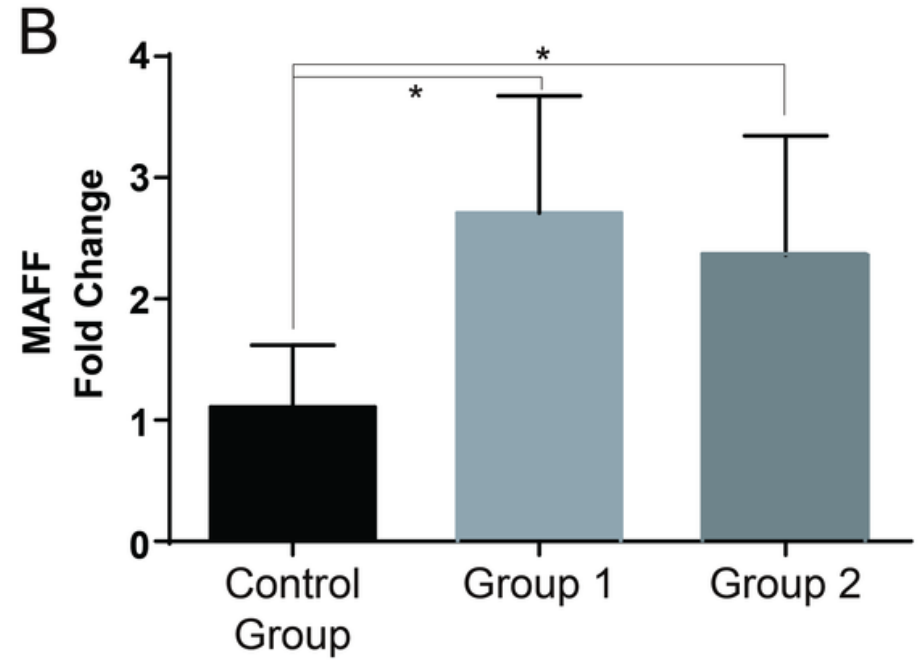

D Control

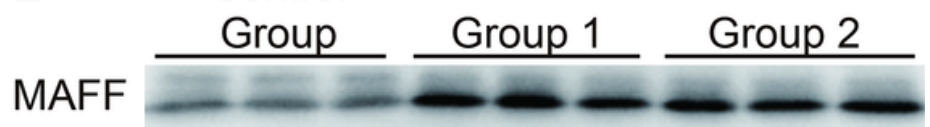
$\beta$-actin

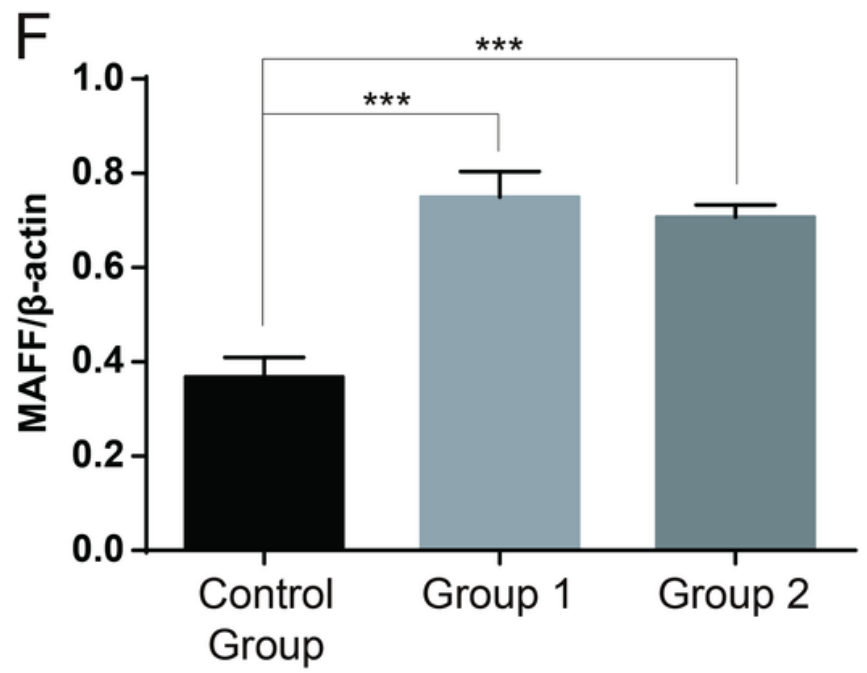

Figure 4

WB and RT-qPCR verification of the two novel candidate key TFs, MAFG and MAFF. (A, B) RT-qPCR validation results. (C, D, E, F) WB validation results. The changing trends of MAFG and MAFF in RT-qPCR and WB results were consistent with the changing trends in TFRE results, so MAFG and MAFF were validated as two novel candidate key TFs in AS. * indicates $0.01<p<0.05$, ** indicates $0.001<p<0.01$, and *** indicates $p<0.001$. 